Recherches en didactique des langues et des cultures

Les cahiers de l'Acedle

14-2 | 2017

Dialogisme et discours en situations didactiques

\title{
Dialogisme et réception des cours magistraux en Français langue étrangère
}

Chantal Parpette et Karine Bouchet

\section{OpenEdition}

Journals

Édition électronique

URL : http://journals.openedition.org/rdlc/1852

DOI : $10.4000 /$ rdlc. 1852

ISSN : 1958-5772

Éditeur

ACEDLE

Référence électronique

Chantal Parpette et Karine Bouchet, « Dialogisme et réception des cours magistraux en Français langue étrangère ", Recherches en didactique des langues et des cultures [En ligne], 14-2 | 2017, mis en ligne le 15 juin 2017, consulté le 19 avril 2019. URL : http://journals.openedition.org/rdlc/1852 ; DOI : $10.4000 /$ rdlc. 1852

Ce document a été généré automatiquement le 19 avril 2019

\section{$\Theta \Theta \Theta \Theta$}

Recherches en didactique des langues et des cultures is licensed under a Creative Commons AttributionNonCommercial-NoDerivatives 4.0 International License 


\title{
Dialogisme et réception des cours magistraux en Français langue étrangère
}

\author{
Chantal Parpette et Karine Bouchet
}

\section{Introduction}

1 Le présent article fait suite à une communication présentée lors d'une journée d'étude sur le dialogisme organisée à l'université Montpellier 3 en octobre 2015. L'objectif de cette communication était, à la lumière des travaux de Bres $(1999,2005)$, d'interroger le dialogisme à l'oeuvre dans le contexte d'enseignements à l'université, et particulièrement au sein des cours magistraux.

2 Le cours magistral est en effet un lieu privilégié de manifestation du dialogisme en ce sens qu'il n'y a pas de dialogue externe, avec tours de parole entre deux ou plusieurs interlocuteurs, mais qu'il y a bien un dialogue interne, une dialogisation dans la structuration du discours de l'enseignant. Celui-ci est le seul locuteur à prendre la parole mais il dialogue avec trois catégories d'interlocuteurs. D'une part, il s'adresse aux étudiants qu'il a en face de lui ; d'autre part, il construit ses données en relation avec les discours antérieurs d'autres chercheurs du domaine; enfin lui arrive de dialoguer avec lui-même. Sa voix est le lieu de rencontre de plusieurs instances d'énonciation, dans une combinaison qui peut être simple, explicite, mais aussi très implicite et complexe (Bouchard \& Parpette, 2007 ; Lepoire-Duc \& Pollet, 2014 ; Carlo \& Claudel, 2014).

Nous nous intéressons ici aux faits de dialogisme dans la perspective de la réception des cours magistraux par les étudiants allophones dans l'université française.

Notre analyse s'organise en deux étapes. La première reprend le cadre théorique proposé par Bres (2005) à partir de discours essentiellement médiatiques, et son application à la description des cours magistraux, telle que la formulent Marlot \& Baques (2013). Nous montrerons dans une deuxième étape quelles modifications nous apportons à ce modèle pour le rendre opérationnel dans le cadre de l'intervention didactique en français langue 
étrangère. L'intervention didactique dont il est question ici sont les activités d'aide à la compréhension des cours magistraux que nous proposons aux étudiants allophones dans le cadre des formations linguistiques destinées à les préparer aux études universitaires en France.

\section{Le dialogisme dans les cours magistraux}

5 S'appuyant sur les travaux de Bakhtine (1984) et les nombreuses recherches qu'ils ont initiées sur le dialogisme, Bres s'est intéressé au phénomène dialogique dans les discours médiatiques. Il distingue trois formes de dialogisme (Bres, 1999, 2005). La première est le dialogisme interdiscursif, présent dans le discours d'un locuteur lorsqu'il fait référence au discours d'un tiers. La deuxième forme est le dialogisme interlocutif qui vise l'allocutaire et ses réactions possibles : le locuteur anticipe sur une réponse plus ou moins prévisible du destinataire de son discours, notamment le lecteur ou l'auditeur des discours médiatiques. A ces deux catégories, il ajoute le dialogisme intralocutif lorsque le locuteur renvoie à son propre discours. Cette catégorie rejoint l'auto-dialogisme d'Authiez-Revuz que celle-ci définit comme un " dédoublement de l'énonciateur » ou encore comme «la non-coïncidence de l'énonciateur à son dire » (1995: 150 et 163).

Marlot et Baques, dans un article de 2013, s'intéressent quant à elles au dialogisme dans les cours magistraux universitaires. Ceux-ci entretiennent avec les discours médiatiques deux différences fondamentales. La première est la présence physique de l'interlocuteur collectif que constitue l'ensemble des étudiants présents dans la salle. Cet interlocuteur ne prend pas la parole mais il fait face à l'enseignant, et il est identifié. Il s'agit par exemple d'un groupe de première année de droit ayant des connaissances, des difficultés, et des réactions relativement connues de l'enseignant, ou dont celui-ci a une représentation. La seconde différence réside dans l'enjeu de la relation locuteurinterlocuteur. L'auteur d'un article a pour objectif d'informer, et souvent de convaincre, quand l'enseignant, lui, vise par son discours à former les étudiants et à les accompagner vers la réussite universitaire. Ces écarts de dispositifs interactionnels induisent d'importantes variations dans les manifestations de dialogisme d'un contexte à l'autre.

Reprenant la tripartition de Bres (2005), Marlot \& Baques (2013 : 7-8) décrivent ainsi le dialogisme dans le cadre du cours magistral :

- Le dialogisme interdiscursif : le locuteur (le professeur) fait référence à des propos qui ne sont pas les siens et les mêle à son discours : on note, d'une manière plus ou moins explicite, la présence de la parole d'autrui dans son discours.

- Le dialogisme intralocutif : le locuteur (le professeur) est son premier interlocuteur. La production de sa parole se fait constamment en interaction avec ce qu'il a dit antérieurement, avec ce qu'il est en train de dire et ce qu'il a à dire.

- Le dialogisme interlocutif : le discours du professeur se construit en considération des étudiants. Cette construction se révèle dans des formes langagières qui prennent en compte l'aspect lexical, syntaxique ou encore la structure argumentative.

8 À partir de ces trois catégories, les deux auteures analysent des passages de deux cours magistraux, un cours de préparation au CAPES de géographie, et un cours d'histoire.

9 La première catégorie, le dialogisme interdiscursif, renvoie aux discours de chercheurs de la discipline enseignée. Ainsi dans l'énoncé : 
Ce système, cette dynamique, permet à l'auteur dont je vous ai parlé tout à l'heure Charles Tilly, de continuer les propos d'Elias, en insistant sur la dynamique guerrière des ÉTATS européens. (Marlot \& Baques, $2013: 13$ )

l'enseignant construit explicitement ce moment de son cours à partir des travaux/ discours de deux historiens de la discipline, Tilly et Elias. Si ce dialogisme interdiscursif est considéré comme présent dans tout discours monologal, le phénomène est particulièrement apparent dans les discours de l'enseignement supérieur qui se construisent dans la filiation-discussion des chercheurs en amont (Bouchard \& Parpette, 2007 ; Claudel, 2010). Nous avons ici un dialogisme très explicite. Nous verrons plus loin qu'il peut revêtir des formes plus implicites et aussi plus impliquées.

Les auteures s'attardent peu sur la seconde catégorie, celle du dialogisme intralocutif, dont elles donnent comme exemples, tirés de leur corpus, les deux énoncés suivants :

(1) Quel que soit l'objet que l'on considère à une certaine échelle on est obligé de voir à quel point les autres échelles sont influentes. (Marlot \& Baques, $2013: 13)$

(2) Donc finalement, ces concurrences militaires... et économiques, elles débouchent sur des oligopoles... inégaux... d'abord, et puis en dernier lieu, sur des monopoles au sein d'un espace géographique qui est toujours déterminé. (op.cit. 13)

11 Nous n'avons pas accès à la totalité de la transcription du passage concerné, mais l'analyse présentée conduit à considérer que ces énoncés sont des conclusions de parties de cours, qui renvoient, comme toute conclusion, à ce qui a été dit précédemment par le locuteur-enseignant lui-même.

12 Ce qui caractérise l'approche de Marlot \& Baques est la très large place qu'elles accordent au dialogisme interlocutif. S'appuyant sur la notion d'anticipation, elles font du dialogisme interlocutif une description très fine et complexe en trois catégories liées à l'Intention $d u$ professeur, elles-mêmes subdivisées en dix Cibles de l'interaction. En voici quelques illustrations : 
Tableau 1 - Extrait de l'analyse du dialogisme interlocutif par Marlot \& Baques (2013)

\begin{tabular}{|c|c|}
\hline $\begin{array}{l}\text { Intention du } \\
\text { professeur }\end{array}$ & Cible de l'interaction - Enoncés \\
\hline \multirow[t]{2}{*}{$\begin{array}{l}\text { 1. anticipation de } \\
\text { la difficulté }\end{array}$} & $\begin{array}{l}\text { 1.1 dans la compréhension de la notion } \\
\text { C'est à dire que Le Roi va donner un territoire à Monsieur X..., et } \\
\text { monsieur X quand il mourra, le territoire va revenir au Roi,... donc, vous } \\
\text { avez pas une perpétuation, euh... de la patrimonialité du territoire }\end{array}$ \\
\hline & $\begin{array}{l}\text { 1.2 dans la compréhension de la structuration du cours } \\
\text { Ce qui fait qu'on va avoir deux sections à traiter : une section sur la } \\
\text { dynamique de l'État moderne, dans laquelle on va parler à la fois de la } \\
\text { genèse de l'État moderne, comme l'État s'est construit... Et puis, dans la } \\
\text { deuxième section, dans la deuxième partie de cette section, on va } \\
\text { réfléchir sur le rôle de la guerre }\end{array}$ \\
\hline \multirow{3}{*}{$\begin{array}{l}\text { 2. aide à } \\
\text { l'imprégnation }\end{array}$} & $\begin{array}{l}2.1 \text { implication directe des étudiants } \\
\text { Est-ce que vous vous rappelez la date? }\end{array}$ \\
\hline & $\begin{array}{l}2.2 \text { référence à une biographie scolaire commune } \\
\text { Voyez le texte de Paul Gardes, si vous l'avez lu, c'était la question de la } \\
\text { minorité, la question de la nationalité, la question gérer la différence et } \\
\text { de construire un État, des choses qu'on a vues ensemble }\end{array}$ \\
\hline & $\begin{array}{l}2.3 \text { référence à une biographie scolaire lointaine } \\
\text { On a tous des automatismes dans notre histoire apprise à l'école }\end{array}$ \\
\hline \multirow{2}{*}{$\begin{array}{l}\text { 3. aide à } \\
\text { l'attention }\end{array}$} & $\begin{array}{l}\text { 3.1 référence à des évidences partagées } \\
\text { Plus on s'approche plus on a de détails ça paraît assez évident }\end{array}$ \\
\hline & $\begin{array}{l}\text { 3.2 création d'un horizon d'attente } \\
\text { Mais est-ce que ça veut dire que l'histoire est déterminée? A votre avis } \\
\text { est-ce que c'était prévu d'avance? }\end{array}$ \\
\hline
\end{tabular}

Compte tenu du face-à-face pédagogique, le dialogisme interlocutif en cours magistral est très souvent combiné aux autres formes de dialogisme, ce que montre bien l'énoncé cité plus haut:

Ce système, cette dynamique, permet à l'auteur dont je vous ai parlé tout à l'heure Charles Tilly... (Marlot \& Baques, $2013: 13$ )

dans lequel l'enseignant se réfère aux travaux de l'auteur ... C. Tilly (interdiscursif) tout en s'adressant directement aux étudiants - je vous... - (interlocutif) pour rappeler son propre discours -j'ai parlé... - (intralocutif). Les différentes formes de dialogisme sont imbriquées, tressées, au point qu'il est difficile d'en isoler les éléments respectifs. On peut dire, comme Bres \& Nowakowska, que le dialogisme interlocutif est pris dans le fonctionnement interdiscursif (2008:9), et vice-versa.

Cette construction du dialogisme interlocutif pose, de notre point de vue, deux questions. En effet, classer un énoncé tel que :

Ce qui fait qu'on va avoir deux sections à traiter: une section sur la dynamique de l'État moderne, dans laquelle on va parler à la fois de la genèse de l'État moderne, comme l'État s'est construit... Et puis, dans la deuxième section, dans la deuxième partie de cette section, on va réfléchir sur le rôle de la guerre. (Marlot \& Baques, $2013: 8$ )

dans la rubrique anticipation sur la difficulté à comprendre la structuration du discours (cf. 1.2 $\mathrm{du}$ tableau) nous semble problématique dans la mesure où cela pose a priori que la dimension essentielle du discours de l'enseignant consiste à lever des difficultés. Il paraît peu légitime d'interpréter toute structuration du discours - annonce du plan ou explication d'une notion - comme un moyen d'éviter un problème de compréhension. 
Nous faisons l'hypothèse d'un transfert peut-être trop fidèle du modèle du dialogisme interlocutif proposé par Bres \& Nowakowska (2008) pour le discours médiatique au cours magistral. Certains discours de presse pratiquent la réponse anticipée à une réaction supposée du lecteur parce que les sujets abordés se prêtent à l'argumentation, voire à la polémique. Il s'agit alors pour l'auteur de l'article de neutraliser à l'avance l'argument contradictoire prévisible chez son lecteur, comme le montre l'exemple suivant :

L'action du gouvernement apparait zigzagante et confuse, même si dans la réalité elle sert toujours les mêmes intérêts. On me dira que ce pouvoir a la faveur de l'opinion. Mais tout pouvoir élu démocratiquement en bénéficie à ses débuts. (Le Monde, 1.2.2003) (op.cit.7)

Il en va tout autrement dans le contexte pédagogique universitaire. Les enjeux du discours enseignant laissent peu de place à ce type dialogisme, l'enseignant étant rarement en situation d'avoir à anticiper sur d'éventuelles objections des étudiants. Cela rend donc problématique ce large périmètre accordé au dialogisme interlocutif et à la notion d'anticipation qui le structure.

La seconde difficulté réside dans la définition fortement sémantisée de certaines cibles de l'interaction: considérer que la référence à une biographie scolaire commune (point $2.2 \mathrm{du}$ tableau ci-dessus) constitue une catégorie différente de la référence à une biographie scolaire lointaine (2.3) revient presque à créer une catégorie par contenu d'énoncé...

\section{Le dialogisme en Français langue étrangère}

17 A partir de ces réflexions, nous nous interrogeons sur la manière dont la didactique du Français langue étrangère peut prendre en compte cette réalité dialogique dans le cadre de la formation linguistique des étudiants allophones.

Comme nous le disions en introduction, l'analyse de Marlot \& Baques nous rapproche de nos préoccupations dans la mesure où elle déplace l'étude du dialogisme des discours médiatiques vers les cours magistraux. Elle en reste néanmoins distincte parce qu'elle s'inscrit dans le champ de l'analyse du discours enseignant et procède à une description de la production alors que, pour notre part, nous nous intéressons au dialogisme pour élaborer des activités d'aide à la compréhension des cours magistraux par les étudiants allophones.

19 Les enjeux de l'intervention didactique en FLE conduisent toujours, quel que soit l'objet de la langue concerné (grammaire, lexique, production écrite, etc.), à une approche simplifiée, "opérationnelle", qui induit une certaine reconfiguration des catégories établies par les sciences du langage. Et comme le disaient en leur temps Beacco \& Darot (1984:115),

Une analyse didactique de discours ne peut pas être une simple expansion des théories déjà constituées en sciences du langage. Elle se doit de présenter un appareil conceptuel économique et une forte puissance descriptive. (c'est nous qui soulignons)

L'analyse que nous proposons opère donc un déplacement de l'enjeu de ces catégories de dialogisme, de la description vers l'intervention didactique : ce n'est plus le dialogisme en tant que tel qui est analysé, mais son impact sur le sens du discours de l'enseignant, et sur sa réception par les étudiants allophones. L'accent est mis :

- sur ce que l'étudiant doit comprendre 
- et sur ce qu'il fait de ce qu'il comprend, en particulier, doit-il le retenir, le prendre en notes?

\section{Vers une reconfiguration des catégories du dialogisme}

Cela nous conduit à traiter le phénomène de dialogisme suivant non pas trois, mais quatre entrées. Pour illustrer cela, nous nous appuyons pour l'essentiel sur un corpus de cours d'économie de première année (Relations monétaires internationales, Economie du développement, Macro-économie), recueilli à l'université Lyon 2.

22 La première entrée est celle du dialogisme interdiscursif : cette référence aux discours antérieurs d'autrui correspond à une catégorie opérationnelle en FLE ne nécessitant aucun déplacement par rapport aux modèles vus dans la première partie.

\section{Exemple 1}

aux Etats-Unis Arthur Lewis / met le doigt sur sur une réalité permanente / hein / ça consiste à dire / que / toute économie et à fortiori les économies du Sud // présentent une double facette (Economie du développement) ${ }^{1}$

4 En revanche, nous proposons un déplacement de catégories des deux autres types de dialogisme - l'interlocutif (prise en considération explicite des destinataires du cours magistral) et l'intralocutif (relation que l'enseignant entretient avec son propre discours) - vers trois entrées distinctes :

- le dialogisme interlocutif, que nous maintenons pour désigner l'adresse directe aux étudiants, en tant que modalité de communication :

\section{Exemple 2}

je vous propose maintenant qu'on interroge les économistes (Economie du développement)

le dialogisme intralocutif, qui comprend les phénomènes de réflexion métaénonciative de l'enseignant sur son propre discours :

donc l'eurodollar c'est / ce qu'on appelle un eurodollar euh ce sont / euh je vais prendre un exemple plutôt que de dire ce sont parce que si je dis ce sont je suis mal barré (Relations monétaires internationales)

\section{Exemple 4}

les relations entre les hommes sont nées avec l'humanité / ça va de soi l'homme n'a jamais été un être unique / lapalissade que je suis en train de proférer là (Introduction au droit)

Il est à noter que ce type de phénomène dialogique n'apparait pas dans l'analyse proposée par Marlot \& Baques (2013), sans doute parce que leur corpus n'en offre pas d'occurrence.

- le dialogisme intradiscursif, que nous ajoutons pour désigner le rappel explicite par l'enseignant de données évoquées précédemment dans son discours :

29 Exemple 5

bien alors petit rappel sur ce qu'on a fait la semaine dernière / encore une fois / ce qu'on cherche à comprendre euh / c'est // bon c'est un phénomène 
dont on a parlé à plusieurs reprises / c'est le phénomène du chômage persistant (Macro-économie) \& Parpette, 2007), il est difficile d'intégrer dans la même catégorie les rappels que fait l'enseignant et les phénomènes de méta-énonciation qui apparaissent lorsque celui-ci interrompt son explication pour faire un commentaire sur une expression qu'il vient d'utiliser. Et si l'étudiant peut trouver intérêt à noter le rappel du cours précédent - sous forme d'un titre, par exemple, parce que cela crée une continuité de contenu - les réflexions méta-énonciatives ne présentent quant à elles aucun intérêt de cet ordre.

Par ailleurs, le phénomène de rappel, largement routinisé dans les cours magistraux, nous semble davantage avoir à faire avec la notion de discours qu'avec celle de locution. Evoquer en début de cours ce que l'on a dit précédemment porte davantage sur le contenu de la parole que sur la forme. C'est la raison pour laquelle il nous semble justifié de parler de dialogisme intradiscursif. Ce découpage rapproche la parole de l'enseignant de celle des autres locuteurs convoqués (interdiscursif), les deux portant sur le contenu. En même temps, la distinction qu'il maintient entre les deux (inter vs intra) correspond à la différence de stratégie de réception-rétention de l'étudiant. Si celui-ci a intérêt à noter le contenu nouveau que constitue la référence aux travaux d'un chercheur cité par l'enseignant, il n'est en revanche pas tenu de noter une seconde fois un discours déjà produit, quel qu'il soit.

Le tableau ci-dessous récapitule les définitions analysées plus haut des différentes catégories de dialogisme, et les déplacements que nous proposons dans la perspective de la réception des cours magistraux.

Tableau 2 - Reconfiguration des catégories de dialogisme suivant une perspective didactique

\begin{tabular}{|c|c|c|c|c|c|}
\hline Bres & $\begin{array}{c}\text { 1. Interdiscursif } \\
\text { Référence aux discours } \\
\text { des autres }\end{array}$ & \multicolumn{2}{|c|}{$\begin{array}{l}\text { 2. Interlocutif } \\
\text { Anticipation sur les discours de } \\
\text { l'allocutaire }\end{array}$} & \multicolumn{2}{|c|}{$\begin{array}{l}\text { 3. Intralocutif } \\
\text { Auto-dialogisme }\end{array}$} \\
\hline Marlot - Baques & $\begin{array}{c}\text { 1. Interdiscursif } \\
\text { Référence aux discours } \\
\text { des autres }\end{array}$ & \multicolumn{2}{|c|}{$\begin{array}{l}\text { 2. Interlocutif } \\
12 \text { catégories } \\
\text { (cf. tableau ci-dessus) }\end{array}$} & \multicolumn{2}{|c|}{$\begin{array}{c}\text { 3. Intralocutif } \\
\text { "Référence à tout ce que dit le locuteur } \\
\text { avant, après, pendant" } \\
\text { Seul le contenu semble traité. Pas } \\
\text { d'évocation d'énoncés méta-énonciatifs }\end{array}$} \\
\hline \multirow[t]{2}{*}{ Parpette - Bouchet } & & $\begin{array}{l}\text { Adresse directe } \\
\text { "Ce système... } \\
\text { dont je vous ai } \\
\text { parlé tout à } \\
\text { l'heure..." }\end{array}$ & $\begin{array}{l}\text { Contenus } \\
\text { «Cest à dire que } \\
\text { Le Roi va donner } \\
\text { un territoire à } \\
\text { Monsieur X...." }\end{array}$ & $\begin{array}{c}\searrow \quad \downarrow \\
\text { Porte sur le } \\
\text { contenu de son } \\
\text { propre discours } \\
\downarrow\end{array}$ & $\begin{array}{l}\text { Porte sur la forme } \\
\text { des mots (énoncé } \\
\text { méta-énonciatif) }\end{array}$ \\
\hline & $\begin{array}{l}\text { 1. Interdiscursif } \\
\text { Référence aux discours } \\
\text { des autres } \\
\text { "aux Etats-Unis Arthur } \\
\text { Lewis / met le doigt sur sur } \\
\text { une réalité permanente... " }\end{array}$ & \multicolumn{2}{|c|}{$\begin{array}{l}\text { 2. Interlocutif } \\
\text { "vous connaissez bien } \\
\text { la } 2^{e} \text { catégorie... " }\end{array}$} & $\begin{array}{l}\text { 3. Intradiscursif } \\
\text { "encore une fois / ce } \\
\text { qu'on cherche à } \\
\text { comprendre euh/ } \\
\text { ''est // bon c'est un } \\
\text { phénomène... " }\end{array}$ & $\begin{array}{l}\text { 4. Intralocutif } \\
\text { "lapalissade que je } \\
\text { suis en train de } \\
\text { proférer là..." }\end{array}$ \\
\hline
\end{tabular}




\section{Le traitement du dialogisme en réception des cours magistraux}

La didactique du FLE appréhende le dialogisme à deux niveaux de l'enseignementapprentissage : celui de la constitution des objets d'enseignement, puis celui des activités de classe.

Le dialogisme interdiscursif est pris en charge comme objet d'enseignement dans certains matériels pédagogiques de Français sur objectif universitaire (FOU) sous le terme de «polyphonie» (Mangiante \& Parpette, 2011; Mangiante \& Raviez, 2015). Le terme de "polyphonie» a été préféré à celui de «dialogisme» parce qu'il indique plus explicitement la présence de plusieurs voix. Par ailleurs, il évite le rapprochement erroné avec le «dialogue externe » et ses tours de parole. Le dialogisme intradiscursif est également pris en charge en tant qu'objet d'enseignement dans les chapitres d'ouvrage intitulés « rappels-annonces ». Ces chapitres sont construits autour de l'écoute d'extraits de débuts de cours magistraux dans lesquels les enseignants rappellent sous des formes très diverses ce qu'ils ont traité lors du cours précédent.

Une fois les objets d'enseignement fixés, le travail de la didactique consiste à construire des activités d'apprentissage permettant aux apprenants d'en acquérir la maitrise. Nous proposons un passage en revue d'activités d'apprentissage permettant le traitement concret de ces faits de dialogisme.

\section{Le dialogisme interdiscursif}

Les faits de dialogisme interdiscursif, dans lesquels l'enseignant fait référence à d'autres discours, sont traités, en situation d'apprentissage du FLE, au moyen d'une analyse guidée d'extraits vidéos, comme dans l'exemple suivant :

\section{Exemple 6 - Économie du développement (vidéo)}

je vous propose maintenant / qu'on interroge les économistes hein sur la question industrielle / qu'on s'intéresse quelques instants aux points de vue de la théorie économique sur l'industrialisation lorsqu'on explore hein le champ de l'analyse économique / il est possible de faire deux tas / hein / c'est-à-dire de regrouper les points de vue proposés en deux catégories // vous avez d'un côté des économistes qui analysent l'industrialisation comme un fait d'organisation // et vous avez par ailleurs des économistes qui analysent / l'industrialisation comme un fait de localisation vous connaissez très bien / la deuxième catégorie d'approche / hein / on l'a également déjà explorée dans le cadre de ce cours / vous connaissez probablement moins / la première catégorie /

je vous propose qu'on passe en revue très brièvement l'analyse / d'Arthur Lewis l'industrialisation duale / ensuite l'analyse d'Oscar Hirschmann l'industrialisation polarisée / et enfin / l'analyse féconde hein de François Perroux en termes de pôles de croissance //chaque fois deux ou trois phrases / seulement hein /

alors tout d'abord Arthur Lewis / dans les années 40- 50 aux Etats-Unis Arthur Lewis / met le doigt sur sur une réalité permanente / hein / ça consiste à dire / que / toute économie et à fortiori les économies du Sud // présentent une double facette mais une double facette qui qui coexiste / en permanence y a d'un côté / la campagne et la ville / hein / la campagne d'un côté la ville de l'autre / hein que l'on peut superposer à secteur traditionnel secteur moderne // et vous pouvez $\mathbf{y}$ aller dans tous les secteurs 
d'activité hein / ville campagne / secteur moderne secteur traditionnel pour Arthur Lewis // l'industrialisation peut être analysée je pense c'est un schéma commode hein / on comprend tout à fait ce que l'auteur veut dire / pour Arthur Lewis / l'industrialisation peut être analysée comme étant / le passage de l'un à l'autre le passage de la campagne vers la ville historiquement c'est bien ça / la mécanisation de l'agriculture donc la hausse de la productivité a eu pour effet un vaste transfert de richesses et de main d'œuvre / de la campagne vers la ville // se faire sur des modalités diverses. Dans un premier temps, l'enseignant fait une présentation «objectivée» (premier paragraphe de la transcription): il signale l'existence de deux catégories d'économistes. Mais il ne s'agit que d'une introduction, qui se poursuit par une imbrication plus complexe entre la voix de l'enseignant et celles des chercheurs qu'il évoque. En effet, il ne s'agit pas pour l'enseignant universitaire de rapporter de manière neutre les discours des autres, mais de les rapporter à travers le filtre de son propre point de vue d'enseignant-chercheur. D'un point de vue didactique, les consignes proposent aux étudiants un repérage en deux temps. Une première consigne :

- Nommez les théories évoquées par l'enseignant dans cet extrait. permet de repérer les trois économistes que l'enseignant se propose d'aborder.

Concernant Oscar Lewis, il s'agit ensuite de guider l'apprenant dans le repérage de sa théorie et de l'évaluation qu'en fait l'enseignant:

- Que dit la théorie d'Oscar Lewis?

- Quelle position exprime l'enseignant vis-à-vis de cette théorie?

- Relevez dans son discours des termes ou expressions qui indiquent sa position.

Cet extrait révèle, en l'occurrence, un florilège d'appréciations positives : analyse féconde, met le doigt sur une réalité, c'est un schéma commode, on comprend tout à fait ce que l'auteur veut dire.

Ceci n'est qu'un exemple, limité, de la diversité des manifestations du dialogisme interdiscursif, et une illustration de ce que la didactique du FLE sait prendre en charge.

D'autres manifestations - pour lesquelles la réflexion didactique reste encore à construire - s'avèrent beaucoup plus complexes, parce qu'elles mettent en jeu, au-delà de la maîtrise des faits linguistiques, une connaissance de l'arrière-plan culturel (Dufour \& Parpette, 2017).

\section{Le dialogisme interlocutif}

Les phénomènes de dialogisme interlocutif présentent généralement peu de difficultés de compréhension pour un auditoire allophone, ces adresses directes de l'enseignant vers les étudiants, telles que je vous propose, vous avez ou vous connaissez, étant aisément repérables. Par ailleurs, elles ne constituent pas un enjeu central dans la compréhension des cours. Lorsque l'on juge cependant utile d'en faire un traitement didactique, ces faits de dialogisme donnent lieu à des consignes de ce type :

Relevez les différentes expressions utilisées par l'enseignant pour entrer en contact avec les étudiants.

Recherches en didactique des langues et des cultures, 14-2 | 2017 
43 soulignés. Notons que ces faits de dialogisme interlocutif présentent un intérêt beaucoup plus grand en expression orale lorsqu'il s'agit de former les étudiants aux exposés par exemple, la réussite de ce type de prestation étant largement liée à la capacité de l'étudiant à créer le contact avec son public (Parpette \& Stauber 2014 : 135-139).

\section{Le dialogisme intradiscursif}

Le traitement didactique du dialogisme intradiscursif consiste quant à lui à faire repérer une habitude langagière présente de manière quasi systématique en début de cours magistral : l'enseignant commence par un bref rappel des derniers contenus abordés (en gras dans la transcription) et en annonçant ceux à venir (en souligné) :

\section{Exemple 7 - Macro-économie}

bon alors / pour aujourd'hui on va euh donc terminer / le chapitre 4 // bien alors petit rappel sur ce qu'on a fait la semaine dernière / euh donc je pense qu'on est dans la partie $1 / 2$ ? // bon 1 partie 1 / je vous rappelle la perspective du chapitre // donc / encore une fois / ce qu'on cherche à comprendre euh / c'est // bon c'est un phénomène dont on a parlé à plusieurs reprises / c'est le phénomène du euh chômage persistant // hein cette idée que quoi qu'on fasse quoi qu'il arrive / depuis les années 70 / on a toujours un taux de chômage qui est euh en général en France en tous cas / supérieur à $8 \%$ // l'approche qu'on a utilisée je vous la rappelle en deux mots puis on va reprendre euh naturellement dans la continuité / c'est de se dire euh que en gros / y a / deux façons d'analyser ce problème / la première on l'a commencée la semaine dernière / la première façon c'est de se dire et de partir d'un postulat / c'est de se dire / c'est à cause du fonctionnement du marché du travail // hein / si depuis 30 ans en France par exemple euh / on a un taux de chômage quand même important / première approche / on était là-dedans il me semble hein / je crois (...)

Les consignes proposent ici un double travail de repérage dans le discours de l'enseignant: le premier porte sur les informations transmises, le second concerne les procédés linguistiques utilisés :

- Écoutez cet extrait et dites brièvement

- ce que rappelle l'enseignant sur le cours précédent

- ce qu'il annonce

- Relevez avec précision les expressions qui indiquent

- un rappel (ex: petit rappel sur)

- une annonce (ex: on va donc terminer)

\section{Le dialogisme intralocutif}

Les phénomènes de dialogisme intralocutif (marques de réflexion méta-énonciative de l'enseignant sur le discours qu'il est en train de produire) sont complexes à percevoir pour les étudiants allophones. Probablement parce qu'ils sont aléatoires et donc non prévisibles. Ils rompent le fil du discours attendu en y introduisant de l'hétérogénéité. Dans l'état actuel de nos réflexions, la stratégie consiste moins à les traiter en compréhension de l'oral qu'à amener les étudiants à prendre conscience de leur existence en travaillant d'abord sur les transcriptions de ces passages de cours : 


\section{Exemple 8 - Relations monétaires internationales}

donc l'eurodollar c'est / ce qu'on appelle un eurodollar euh ce sont / euh je vais prendre un exemple plutôt que de dire ce sont parce que si je dis ce sont je suis mal barré/ imaginez que vous êtes un entrepreneur japonais et que...

\section{Exemple 9 - Introduction au droit}

on peut conclure de cette échappée historique que je viens de faire dans le temps hein / on peut conclure inévitablement que / enfin / inévitablement / avec peu de chance de se tromper disons tout de même parce qu'il n'y a pas de preuve scientifique encore une fois / on peut conclure avec peu de chance donc de faire erreur que euh le droit est apparu en même temps que les relations sociales / les relations entre les hommes sont nées avec l'humanité / ça va de soi l'homme n'a jamais été un être unique / lapalissade que je suis en train de proférer là / donc dès que la première société s'est formée il y a fort à parier pour que elle ait eu recours à des premières formes de droit des premières formes d'organisation juridique de ses relations internes / et de ses relations aussi extérieures/

Ces énoncés (en gras ci-dessus) proférés par l'enseignant sur son propre discours immédiat mettent en évidence ce que Authier-Revuz (1995) présente à travers la notion de non-coïncidence entre les mots et les choses, et introduisent un double niveau de discours. Le discours passe de la transmission d'un contenu - la définition de l'euro-dollar dans le premier exemple, ou la genèse des relations sociales dans le second - à un commentaire sur les mots, se déplaçant ainsi du contenu vers la forme. Il faut une très bonne réactivité en compréhension pour ne pas perdre le fil de cette construction discursive qui complexifie notablement la réception pour des étudiants allophones.

51 Les consignes de compréhension conduisent l'étudiant à repérer les commentaires que l'enseignant porte sur son propre discours, puis à cerner en quoi ces commentaires viennent modifier ou nuancer ce discours. Par exemple :

- Quelle est la fonction des énoncés en gras?

- Comment modifient-ils les explications qui précèdent?

Dans le premier exemple, il s'agira de relever que l'enseignant passe de l'explication de l'euro-dollar par une définition à une explication par un exemple, ce qui lui paraît plus simple. Dans le second, l'enseignant nuance une affirmation quelque peu catégorique ( inévitablement), et souligne plus loin une remarque un peu inutile parce que relevant de l'évidence.

53 Nous avons souligné plus haut que le traitement didactique du dialogisme touchait non seulement la compréhension du discours de l'enseignant mais également la rétention des données. L'étudiant doit en effet garder une trace matérielle de ses cours et, dans la majorité des cas, il s'agit d'une prise de notes. Or l'étape première et essentielle de la prise de notes réside dans la sélection des données à conserver. Les faits de dialogisme analysés ici sont largement concernés par cette préoccupation de la prise de notes. On peut en effet considérer que le dialogisme interdiscursif, très présent dans les disciplines socialement impliquées telles que l'économie ou la science politique, s'inscrit dans les données fondamentales du cours et appelle la prise de notes. A l'opposé, le dialogisme interlocutif, tel que nous l'avons défini, n'est que l'habillage oral de la communication en face-à-face et ne relève en rien des données du cours. Le dialogisme intralocutif, tel qu'il 
apparaît dans nos exemples, s'apparente à des digressions, des nuances qui sont généralement laissées de côté lors de la prise de notes. Quant au dialogisme intradiscursif, la stratégie dépend davantage de chaque étudiant-noteur: certains jugent inutile de reprendre des données déjà traitées, d'autres, recourant à une stratégie moins sélective, ou plus rassurante, préfèrent noter à nouveau.

\section{Conclusion}

Les exemples présentés ici n'épuisent pas la grande diversité des manifestations du dialogisme entre les différentes instances d'énonciation présentes dans le discours d'un enseignant. Entre référence très explicite et allusion, entre présentation objectivée et implication approbatrice ou ironique, on passe d'un dialogisme clairement repérable et interprétable à un dialogisme diffus ou à une imbrication complexe de voix multiples. Dans le cadre des cours magistraux en FLE, nous nous intéressons essentiellement au dialogisme qui joue un rôle dans les contenus disciplinaires que veut transmettre l'enseignant et que l'étudiant doit comprendre pour interpréter correctement les données reçues. Cela donne une place centrale au dialogisme interdiscursif, constitutif de la démarche d'élaboration et de diffusion des savoirs à l'université. Ce choix stratégique clarifié, il reste à approfondir les procédures didactiques susceptibles d'accompagner les étudiants allophones dans la maîtrise des formes complexes de ce dialogisme.

\section{BIBLIOGRAPHIE}

Authier-Revuz, J. (1995). Ces mots qui ne vont pas de soi. Boucles réflexives et non-coïncidences du dire. Paris : Larousse.

Bakhtine, M. (1952/1984). « Les genres du discours », In Esthétique de la création verbale. Paris : Gallimard, pp. 265-308.

Beacco, J.-C. \& Darot, M. (1984). Analyses de discours, lecture et expression, Paris : Hachette-Larousse.

Bouchard, R. \& Parpette, C. (2007). « Autoportrait de l'enseignant-chercheur en auteur/acteur. Jeu de postures et reformulations dans les cours magistraux de $1^{\text {re }}$ année ». LIDIL. Revue de linguistique et de didactique des langues, $\mathrm{n}^{\circ} 35$. pp. 119-136.

Bres, J. (1999). « Entendre des voix : de quelques marqueurs dialogiques en français ». In Bres, J., Delamotte-Legrand, R., Madray-Lesigne, F. \& Siblot, P. (éds). L'Autre en discours, Montpellier :

PUM. pp. 191-212.

Bres, J. (2005). « Savoir de quoi on parle : dialogue, dialogal, dialogique ; dialogisme, polyphonie... ». In Bres, J. et al. Dialogisme et polyphonie. Louvain : De Boeck supérieur. pp. 47-61.

Bres, J. \& Nowakowska, A. (2008). « J'exagère ! ... Du dialogisme interlocutif ». In Birkelund, M., Mosagaard Hansen, M.B. \& Norén, C. (éds). L'énonciation dans tous ses états. Bruxelles : Peter Lang. pp. 1-27. 
Carlo, C. \& Claudel, C. (2014). « Procédés discursifs saillants dans les cours magistraux et stratégies d'enseignement en français en contexte universitaire ». In Cours magistraux et environnements numériques : stratégies des enseignants, stratégies des étudiants. Laboratoire ICAR, Journée d'étude, 17 octobre 2014. http://icar.univ-lyon2.fr/pages/documents/Carlo-Claudel.pdf.

Claudel, C. (2010). « Pratiques citationnelles dans des cours magistraux à l'université ». Ci-Dit, Communications du IV $V^{e} i$-dit, Pratiques citationnelles dans des cours magistraux à l'université. http:// revel.unice.fr/symposia/cidit/index.html?id=423.

Dufour, S. \& Parpette, C. (2017). « Le cours magistral : interrogations didactiques et analyse de discours ». Les carnets du CEDISCOR. Paris : Presses de la Sorbonne Nouvelle (sous presse).

Lepoire-Duc, S. \& Pollet, M. C. (2014). « Les procédés d'orchestration du cours : discours oral, gestualité et supports écrits ». In Cours magistraux et environnements numériques: stratégies des enseignants, stratégies des étudiants. Laboratoire ICAR, Journée d'étude, 17 octobre 2014. http:// icar.univ-lyon2.fr/pages/documents/Lepoire-Pollet.pdf.

Mangiante, J.-M. \& Parpette, C. (2011). Le français sur objectif universitaire. Grenoble : PUG.

Mangiante, J.-M. \& Raviez, F. (2015). Réussir ses études littéraires en français. Grenoble : PUG.

Marlot, C. \& Baques, M.C. (2013). « Le dialogisme à l'œuvre dans le cours magistral à l'université : un point de vue didactique ». In Apprendre et former : la dimension langagière, Presses Universitaires Blaise-Pascal. www.lcdpu.fr. pp. 359-394, Sphère éducative, 978-2-84516-584-7. <halshs-00976327>.

Parpette, C. \& Stauber, J. (2014). Réussir ses études d'économie-gestion en français. Grenoble : PUG.

\section{NOTES}

1. Convention de transcription : / indique une pause brève, // indique une pause plus longue.

\section{RÉSUMÉS}

Le dialogisme, largement présent dans les cours magistraux de sciences humaines, peut, dans ses manifestations les plus complexes, poser des problèmes de compréhension aux étudiants allophones. A partir des travaux de Bres (2005), et de l'application à la description du cours magistral qu'en proposent Marlot \& Baques (2013), l'article interroge les phénomènes dialogiques dans une perspective d'intervention en FLE. En étudiant un corpus de cours d'économie recueilli au sein de l'université Lyon 2, nous nous penchons sur la manière dont la didactique du FLE peut s'emparer de ce fait de discours pour proposer des d'activités de compréhension des cours magistraux par les étudiants allophones.

Dialogism, widely present in human sciences lectures can, in its most complex manifestations, cause comprehension problems for non-native speakers. From the work of Bres (2005), and its application to the description of lectures by Marlot \& Baques (2013), the article questions the dialogical phenomena from an interventionist perspective on teaching and learning French as a foreign language. By studying a corpus of economics courses collected at the University Lyon 2, 
we analyse how French foreign language didactics can take hold of this discourse phenomenon to develop lecture comprehension activities by non-native students.

\section{INDEX}

Mots-clés : dialogisme, cours magistraux, français langue étrangère, étudiants allophones

Keywords : dialogism, lectures, french as a foreign language, non-native students

\section{AUTEURS}

\section{CHANTAL PARPETTE}

Université Lumière-Lyon 2 - Laboratoire ICAR - UMR 5191

Chantal Parpette est Maîtresse de conférences en Didactique du FLE (département des Sciences du langage) à l'Université Lumière-Lyon 2. Elle assure en Master FLE des enseignements de méthodologie du FLE, français sur objectif spécifique, didactique l'oral, interactions didactiques, et met en place des formations extérieures auprès d'enseignants de FLE. Ses recherches actuelles portent sur le français sur objectif universitaire. Elle est l'auteure de matériel pédagogique pour les apprenants de FLE et d'ouvrages de formation pour les enseignants.

chantal.parpette [at] univ-lyon2.fr

\section{KARINE BOUCHET}

Université Lumière-Lyon 2 - Laboratoire ICAR - UMR 5191

Karine Bouchet est doctorante en Didactique du FLE (département des Sciences du langage) à l'Université Lumière-Lyon 2, sous la direction de Chantal Parpette et Catherine Carlo. Ses recherches portent sur l'enseignement-apprentissage du français sur objectif universitaire, en particulier sur les questions de réception des cours magistraux et de prise de notes par des étudiants allophones suivant des modalités collaboratives. Elle est également enseignante de FLE à l'ILCF de l'université catholique de Lyon, et à l'Institut d'Études Politiques de Lyon. karine.bouchet1 [at] univ-lyon2.fr 